\title{
Dochead thérapeutique
}

Sous-dochead gastro-entérologie

\section{Les médicaments de la diarrhée}

\section{Jacques Buxeraud ${ }^{\mathrm{a}, *}$}

Professeur émérite des Universités

\section{Sébastien Faure ${ }^{b}$}

Professeur des Universités

\section{Didier Denardou ${ }^{c}$}

Préparateur en pharmacie, directeur du CFA de la pharmacie de Limoges

${ }^{a}$ Faculté de pharmacie, 2 rue du Docteur-Marcland, 87025 Limoges cedex, France

${ }^{\mathrm{b}}$ Faculté de santé, Département pharmacie, Université d'Angers, 16 boulevard Daviers, 49045 Angers, France

${ }^{c}$ Espace Galien, 40-42 rue du Mas-Loubier, 87100 Limoges, France

\section{*Auteur correspondant.}

Adresse e-mail : jacques.buxeraud@unilim.fr (J. Buxeraud).

\section{Résumé}

De nombreux médicaments sont à notre disposition pour traiter les diarrhées. La plupart sont bénignes et ne nécessitent pas de consultation médicale. En observant des règles hygiénodiététiques et une thérapeutique symptomatique simples, elles doivent rapidement s'estomper.

(C) 2019

Mots clés - antidiarrhéiques ; diarrhée ; lopéramide ; médicaments ; racécadotril 


\section{Keywords à venir}

La diarrhée est un problème digestif fréquent qui se caractérise par des selles de consistance liquide ou molle, plus volumineuses et nombreuses qu'à l'habitude (plus de trois par jour). Elle est dite "aiguë" lorsqu'elle apparaît brutalement et qu'elle évolue sur une durée de quelques jours seulement. Les principales thérapeutiques disponibles sont les ralentisseurs du transit intestinal, les antisécrétoires intestinaux, les produits d'origine microbienne, les topiques adsorbants, les antiseptiques intestinaux et les solutions de réhydratation orale (SRO) [1,2].

\section{T1 Le lopéramide, ralentisseur du transit intestinal}

TEG1 Le lopéramide est l'antidiarrhéique le plus connu mais aussi l'un des plus efficaces, grâce à son effet antisécrétoire et sa faculté à ralentir le transit colique. D'action très rapide et durable, il est indiqué dans le traitement symptomatique des diarrhées aiguës.

TEG1 II importe de respecter les doses conseillées et de veiller à ce que le traitement ne soit pas trop prolongé car ce médicament a tendance, en ralentissant le transit, à maintenir le germe en cause dans la lumière intestinale. Par ailleurs, il n'est pas dénué d'effets indésirables (constipation, douleurs abdominales, ballonnements, nausées, vomissements, sécheresse buccale, réactions d'hypersensibilité, asthénie, somnolence, vertiges, rares cas de rétention urinaire).

TEG1 Diverses présentations hors liste sont disponibles (tableau 1). La posologie initiale est de $2 \mathrm{mg}$, puis $2 \mathrm{mg}$ supplémentaires, après chaque selle non moulée. Il convient de ne jamais prendre plus de six gélules, six comprimés ou six lyophilisats par jour et de ne pas dépasser deux jours de traitement.

TEG1 Le lopéramide peut aussi être aussi prescrit par un médecin dans la diarrhée chronique, à raison d'une à trois gélules par jour chez l'adulte et d'une à deux gélules par jour chez l'enfant [1].

Tableau 1. Spécialités à base de lopéramide.

\begin{tabular}{llll}
\hline & Spécialités & Présentation & Indications \\
\hline $\begin{array}{l}\text { Conseil à } \\
\text { l'officine (hors }\end{array}$ & Diarétyl $^{\circledR}$ & Cp 2 mg (boîte de 12) & $\begin{array}{l}\text { Adultes et enfants } \\
\text { à partir de 15 ans }\end{array}$ \\
liste) & Diastrolib $^{\circledR}$ & $\begin{array}{l}\text { Lyophilisat oral 2 mg } \\
\text { (boîte de 10) }\end{array}$ &
\end{tabular}




\begin{tabular}{|c|c|c|c|}
\hline & Ercestop ${ }^{\circledR}$ & Gél 2 mg (boîte de 12) & \\
\hline & $\begin{array}{l}\text { Gastrowell } \\
\text { lopéramide }^{\circledR}\end{array}$ & Gél 2 mg (boîte de 12) & \\
\hline & Imodiumcaps ${ }^{\circledR}$ & Gél 2 mg (boîte de 12) & \\
\hline & Imodiumlingual ${ }^{\circledR}$ & $\begin{array}{l}\text { Lyophilisat oral } 2 \mathrm{mg} \\
\text { (boîte de 12) }\end{array}$ & \\
\hline & $\begin{array}{l}\text { Imodiumliquicap } \\
\mathrm{s}^{\circledast}\end{array}$ & $\begin{array}{l}\text { Caps molle } 2 \mathrm{mg} \\
\text { (boîte de 12)) }\end{array}$ & \\
\hline & Indiaral ${ }^{\circledR}$ & Gél 2 mg (boîte de 12) & \\
\hline & Imodiumduo ${ }^{\circledR}$ & $\begin{array}{l}\text { Cp } 2 \text { mg, associé à } 125 \text { mg } \\
\text { de siméticone (boîte de 8) }\end{array}$ & $\begin{array}{l}\text { Adultes et enfants } \\
\text { à partir de } 12 \text { ans }\end{array}$ \\
\hline Sur prescription & Arestal ${ }^{\circledR}$ & Cp 1 mg (boîte de 20) & Adultes \\
\hline (liste II) & Imodium $^{\circledast}$ & Gél (boîte de 20) & $\begin{array}{l}\text { Adultes et enfants } \\
\text { à partir de } 8 \text { ans }\end{array}$ \\
\hline & Imodium $^{\circledR}$ & $\begin{array}{l}\text { Sol buv pour enfant } \\
0,2 \mathrm{mg} / \mathrm{mL} \text { (flacon de } 90 \mathrm{~mL} \text { ) }\end{array}$ & $\begin{array}{l}\text { Enfants de } 2 \text { à } \\
8 \text { ans }\end{array}$ \\
\hline
\end{tabular}

De nombreux génériques sont disponibles hors liste pour le conseil et en liste II.

Sur 3 colonnes ou 2 colonnes + marge près de son appel

\section{T1 Le racécadotril, antisécrétoire intestinal}

TEG1 Le racécadotril est efficace et d'action rapide dans le traitement symptomatique des diarrhées aiguës. Il diminue l'hypersécrétion intestinale d'eau et d'électrolytes, mais ne modifie pas le temps de transit intestinal [3].

TEG1 Certaines formes de racécadotril hors liste peuvent dorénavant faire partie du conseil officinal (tableau 2).

TEG1 Chez l'adulte, il s'administre à raison, par exemple, d'une gélule à $100 \mathrm{mg}$ d'emblée, quel que soit le moment, puis d'une gélule trois fois par jour, de préférence au début des trois principaux repas. Le traitement est poursuivi jusqu'à l'obtention de deux selles moulées, sans jamais dépasser sept jours de traitement.

TEG1 Chez l'enfant, le racécadotril 30 mg est indiqué pour un poids supérieur ou égal à $13 \mathrm{~kg}$. 
TEG1 Chez le nourrisson à partir d'un mois et de moins de $13 \mathrm{~kg}$, le dosage à $10 \mathrm{mg}$ est utilisé. La posologie usuelle journalière est établie en fonction du poids corporel sur la base de $1,5 \mathrm{mg} / \mathrm{kg}$ par prise, avec une prise d'emblée, puis trois prises réparties dans la journée. Le traitement doit être poursuivi jusqu'au retour de deux selles moulées, sans dépasser sept jours.

Tableau 2. Spécialités à base de racécadotril.

\begin{tabular}{|c|c|c|c|}
\hline & Spécialités & Présentation & Liste \\
\hline \multirow{3}{*}{$\begin{array}{l}\text { Conseil à } \\
\text { l'officine }\end{array}$} & $\operatorname{Diarfix}^{\circledast}$ & Gél à 100 mg (boîte de 10) & Hors liste \\
\hline & & & \\
\hline & Tiorfast $^{\circledR}$ & Gél à 100 mg (boîte de 10) & Hors liste \\
\hline \multirow{7}{*}{$\begin{array}{l}\text { Sur } \\
\text { prescription } \\
\text { médicale }\end{array}$} & Tiorfan $^{\circledR}$ & Gél à 100 mg (boîte de 20) & Liste II \\
\hline & & & \\
\hline & & Poudre enfant $30 \mathrm{mg}$ & Liste I \\
\hline & & (boîte de 30 sachets) & \\
\hline & & Poudre enfant $10 \mathrm{mg}$ & Liste I \\
\hline & & (boîte de 16 sachets) & \\
\hline & Tiorfanor $^{\circledR}$ & $\begin{array}{l}\text { Comprimé pelliculé } 175 \text { mg } \\
\text { (boîte de 12) }\end{array}$ & Liste II \\
\hline
\end{tabular}

De nombreux génériques sont disponibles hors liste pour le conseil et en liste II.

Sur 3 colonnes ou 2 colonnes + marge près de son appel

\section{T1 Les produits d'origine microbienne}

TEG1 Ultra-Levure ${ }^{\circledR}$ est une flore de substitution à base levures Saccharomyces boulardii indiquée dans le traitement symptomatique d'appoint de la diarrhée [4] :

- la forme gélule est réservée à l'adulte et à l'enfant de plus de 6 ans ; le dosage à $50 \mathrm{mg}$ s'utilise à la dose de quatre gélules par jour en deux prises et le dosage à $200 \mathrm{mg}$ à raison d'une gélule par jour ;

- la forme poudre pour suspension buvable en sachet de $100 \mathrm{mg}$, réservée à l'adulte et à l'enfant de plus de 2 ans, s'administre à la posologie de deux sachets par jour, en deux prises.

Du fait de sa nature fongique, Ultra-Levure ${ }^{\circledR}$ ne doit pas être associé à un antifongique oral ou systémique.

TEG1 Lenia ${ }^{\circledR}$ appartient au groupe des micro-organismes antidiarrhéiques à base de Lactobacillus casei variété rhamnosus : la forme gélule à $250 \mathrm{mg}$ est réservée à l'adulte (deux à huit gélules par 
jour) et la forme suspension buvable en sachet-dose à 1,5 g à l'enfant de plus de 2 ans (un à quatre sachets-dose par jour).

TEG1 Lactéol ${ }^{\circledR}$ renferme des germes inactivés de Lactobacillus (Lactobacillus fermentum et delbrueckii). Les substances actives sont les produits métaboliques élaborés par les Lactobacillus inactivés après culture dans un milieu à base de lactose. Ce médicament se présente sous la forme de gélule (réservée à l'adulte et à l'enfant de plus de 6 ans) et de poudre pour suspension buvable qu'il est possible d'utiliser même chez le nourrisson. Bactériostatique et immunostimulant au niveau des muqueuses, Lactéol ${ }^{\circledR}$ favorise également la croissance de la flore acidogène de défense, principalement grâce à la présence de vitamines du groupe $B$.

Ce traitement symptomatique d'appoint de la diarrhée est indiqué en complément de la réhydratation et/ou des mesures diététiques, à raison d'une à deux gélules par jour, en fonction de l'intensité des troubles, mais jusqu'à trois gélules le premier jour de traitement.

Les patients ayant des antécédents d'allergie aux protéines de lait de vache ne doivent pas prendre ce médicament qu'il est préférable de ne pas utiliser également pendant la grossesse et l'allaitement.

\section{T1 Les topiques adsorbants}

TEG1 Grâce à leur action barrière et leur capacité de fixation élevée, les topiques adsorbants protègent la muqueuse digestive agressée. Ils donnent souvent de bons résultats et ont l'avantage d'être pratiquement dénués d'effets indésirables, en dehors du risque de constipation. Toutefois, les propriétés adsorbantes de ces produits peuvent interférer avec les délais et/ou les taux d'absorption d'une autre substance prise par le patient. Il est donc recommandé d'administrer tout autre médicament à distance.

TEG1 Actapulgite ${ }^{\circledR}$ (attapulgite de Mormoiron activée) est indiqué dans le traitement symptomatique des manifestations fonctionnelles intestinales, notamment avec météorisme et diarrhée. Ce médicament s'utilise, de préférence avant les repas, à raison de deux à trois sachets par jour chez l'adulte et de deux sachets par jour chez l'enfant de plus de $10 \mathrm{~kg}$. Afin d'obtenir un mélange parfaitement homogène et de goût agréable, il est recommandé de mélanger à sec ce médicament avec du sucre en poudre avant adjonction d'eau.

TEG1 Smecta ${ }^{\circledR}$ (diosmectite) possède un pouvoir couvrant important de la muqueuse digestive (tableau 3).

Tableau 3. Médicaments à base de diosmectite dans la diarrhée aiguë.

\begin{tabular}{lll}
\hline Spécialités & Forme et dosage & Posologie et administration $^{1}$ \\
\hline Smecta $^{\circledR}$ & $\begin{array}{l}\text { Poudre pour susp } \\
\text { buv à } 3 \mathrm{~g} \text { en sachet }\end{array}$ & $\begin{array}{l}\text { Enfant à partir de } 2 \text { ans : } 4 \text { sachets/jour pendant trois } \\
\text { jours, puis } 2 \text { sachets/jour pendant quatre jours }\end{array}$ \\
& & \\
\end{tabular}


fraise (boîte de 12) Le contenu du sachet peut être délayé dans un biberon de $50 \mathrm{~mL}$ d'eau à répartir au cours de la journée ou

Poudre pour susp

buv à $3 \mathrm{~g}$ en sachet

orange vanille mélangé à un aliment semi-liquide (bouillie, compote, purée, etc.)

(boîte de 30 et

Adulte : 3 sachets/jour pendant sept jours (la posologie de 60) peut être doublée en début de traitement)

Le contenu du sachet peut être délayé dans un demiverre d'eau

\begin{tabular}{lll}
\hline Smectalia $^{\circledR}$ & $\begin{array}{l}\text { Poudre pour susp } \\
\text { buv à } 3 \text { g en sachet } \\
\text { (boîte de 18) }\end{array}$ & $\begin{array}{l}\text { Adultes et enfants de plus de 15 ans : 1 sachet, puis } \\
\text { 1 sachet après chaque nouvelle selle non moulée, sans } \\
\text { dépasser } 6 \text { sachets/jour (durée maximale de traitement } \\
\text { de trois jours) }\end{array}$ \\
$\begin{array}{l}\text { en sachet } \\
\text { (boîte de 12) }\end{array}$ & $\begin{array}{l}\text { Poudre pour suspension buvable : le contenu du sachet } \\
\text { doit être mis en suspension juste avant utilisation, par } \\
\text { exemple dans un demi-verre d'eau }\end{array}$ \\
& $\begin{array}{l}\text { Suspension buvable : pour fluidifier la suspension, il est } \\
\text { recommandé de malaxer le sachet entre les doigts avant } \\
\text { de l'ouvrir }\end{array}$
\end{tabular}

${ }^{1}$ De préférence à distance des repas.

Sur 3 colonnes ou 2 colonnes + marge près de son appel

\section{T1 Les antiseptiques intestinaux}

TEG1 Le nifuroxazide (Ercéfuryl ${ }^{\circledR}$ ) est un antibactérien intestinal proche des antibiotiques, disponible hors liste. Son indication est toutefois limitée : "diarrhée aiguë présumée d'origine bactérienne en l'absence de suspicion de phénomènes invasifs (altération de l'état général, fièvre, signes toxi-infectieux) ». Cet antibactérien peut altérer le microbiote et donc aggraver la situation. Les gastro-entérologues s'accordent pour dire qu'il ne devrait plus être utilisé.

TEG1 Quant aux antibiotiques (quinolones, azithromycine, céphalosporines de troisième génération, etc.), ils ne peuvent être délivrés que sur prescription médicale, dans des situations ciblées soigneusement appréciées par le médecin : diarrhées de type invasif, terrains à risques, diarrhées dues aux antibiotiques, etc.

\section{T1 Les solutions de réhydratation orale}


TEG1 Au cours d'un épisode de diarrhée aiguë, l'organisme perd souvent beaucoup d'eau et de sels minéraux (sodium, potassium, etc.). Il est nécessaire de compenser le plus rapidement possible ces pertes en buvant le plus souvent possible des boissons salées et sucrées (eau simple ou sucrée, sodas, bouillon de légumes, infusions). Cette réhydratation est d'autant plus importante que le sujet est très jeune ou âgé.

TEG1 Les SRO constituent le traitement essentiel de toute diarrhée aiguë. Adiaril ${ }^{\circledR}$ est destiné à assurer par voie orale la compensation des pertes en minéraux et en eau consécutives aux diarrhées et gastro-entérites aiguës infantiles. Le sachet est dilué dans $200 \mathrm{~mL}$ d'eau faiblement minéralisée, sans adjonction de sucre ni de sel. La solution est administrée par voie orale à température ambiante. La quantité est déterminée par le médecin en fonction des besoins hydriques de l'enfant, dès l'apparition de la diarrhée ou des signes de déshydratation. Cette SRO sera proposée à volonté pendant les premières heures à intervalles réguliers, toutes les cinq à dix minutes au début, puis toutes les quinze à trente minutes.

\section{T1 Les produits divers}

TEG1 Diaralia ${ }^{\circledR}$ est un médicament homéopathique, disponible sous la forme de comprimés à $300 \mathrm{mg}$, à base d'Arsenicum album $9 \mathrm{CH}$, China rubra $5 \mathrm{CH}$ et Podophyllum peltatum $9 \mathrm{CH}$. Il est traditionnellement utilisé dans le traitement de courte durée des diarrhées aiguës passagères chez l'adulte et l'enfant de plus de 6 ans, à raison d'un comprimé à sucer, quatre à six fois par jour durant trois jours au maximum. Son usage doit être évité pendant la grossesse.

TEG1 Elusanes Salicaire ${ }^{\circledR}$ renferme de l'extrait sec de salicaire, sous la forme de gélules. Ce produit est indiqué dans le traitement symptomatique des diarrhées légères chez l'adulte et l'adolescent de plus de 12 ans à la posologie de deux gélules, deux à trois fois par jour. La durée maximale de traitement est de deux jours. Si les symptômes persistent au-delà, un médecin doit être consulté. L'utilisation pendant la grossesse et l'allaitement est déconseillée.

TEG1 Salicairine ${ }^{\circledR}$ est une solution buvable à base d'extrait fluide de salicaire qui peut être utilisée comme traitement symptomatique dans les diarrhées à la posologie de 30 à 60 gouttes par prise, trois fois par jour. Il est préférable de ne pas utiliser ce médicament pendant la grossesse.

TEG1 L107 ${ }^{\circledR}$ est un médicament homéopathique traditionnellement utilisé dans le traitement adjuvant des gastro-entérites aiguës, disponible sous la forme de comprimés orodispersibles et de solution buvable en gouttes.

\section{Les points à retenir}

- Le lopéramide, d'action très rapide et durable, est indiqué dans le traitement symptomatique des diarrhées aiguës. 
- Le racécadotril est un antisécrétoire intestinal efficace et d'action rapide dans le traitement symptomatique des diarrhées aiguës.

- Saccharomyces boulardii est une levure indiquée dans le traitement symptomatique d'appoint de la diarrhée.

- Les topiques adsorbants protègent la muqueuse digestive agressée. Ils ne présentent pratiquement pas d'effets indésirables, en dehors du risque de constipation.

- Les solutions de réhydratation orale (SRO) constituent le traitement essentiel de toute diarrhée aiguë.

\section{Sur deux colonnes à droite après la puce de fin}

Déclaration de liens d'intérêts

Les auteurs déclarent ne pas avoir de liens d'intérêts.

\section{Références}

[1] Vidal. Les traitements de la diarrhée chez l'adulte.

https://eurekasante.vidal.fr/maladies/estomac-intestins/diarrhee-adulte.html?pb=traitements

[2] Pharmacomédicale.org. Antidiarrhéiques : les points essentiels.

https://pharmacomedicale.org/medicaments/par-specialites/item/antidiarrheiques-les-pointsessentiels

[3] Haute Autorité de santé (HAS). Commission de la Transparence. Racécadotril. Avis du 22 juin 2016. www.has-sante.fr/portail/upload/docs/evamed/CT-14259_TIORFANTIORFANOR_PIS_RI_Avis1_CT14259.pdf

[4] Vidal. Ultra-Levure. https://eurekasante.vidal.fr/medicaments/vidal-famille/medicamentbultra01-ULTRA-LEVURE.html

\section{Illustrations}

\section{Buxera_illus1}

La diarrhée se caractérise par des selles de consistance liquide ou molle, plus nombreuses qu'à I'habitude, lorsqu'elle apparaît brutalement et évolue sur quelques jours seulement, elle est dite "aiguë".

(C) princeoflove/stock.adobe.com 
En première page sur 1 colonne en haut à droite

Buxera_illus2

Les solutions de réhydratation orale constituent le traitement essentiel de toute diarrhée aiguë chez l'enfant, en compensant les pertes en minéraux et en eau consécutives aux diarrhées et gastroentérites aiguës.

(c) wendyhayesrise/stock.adobe.com

En dernière page sur 1 colonne en haut 


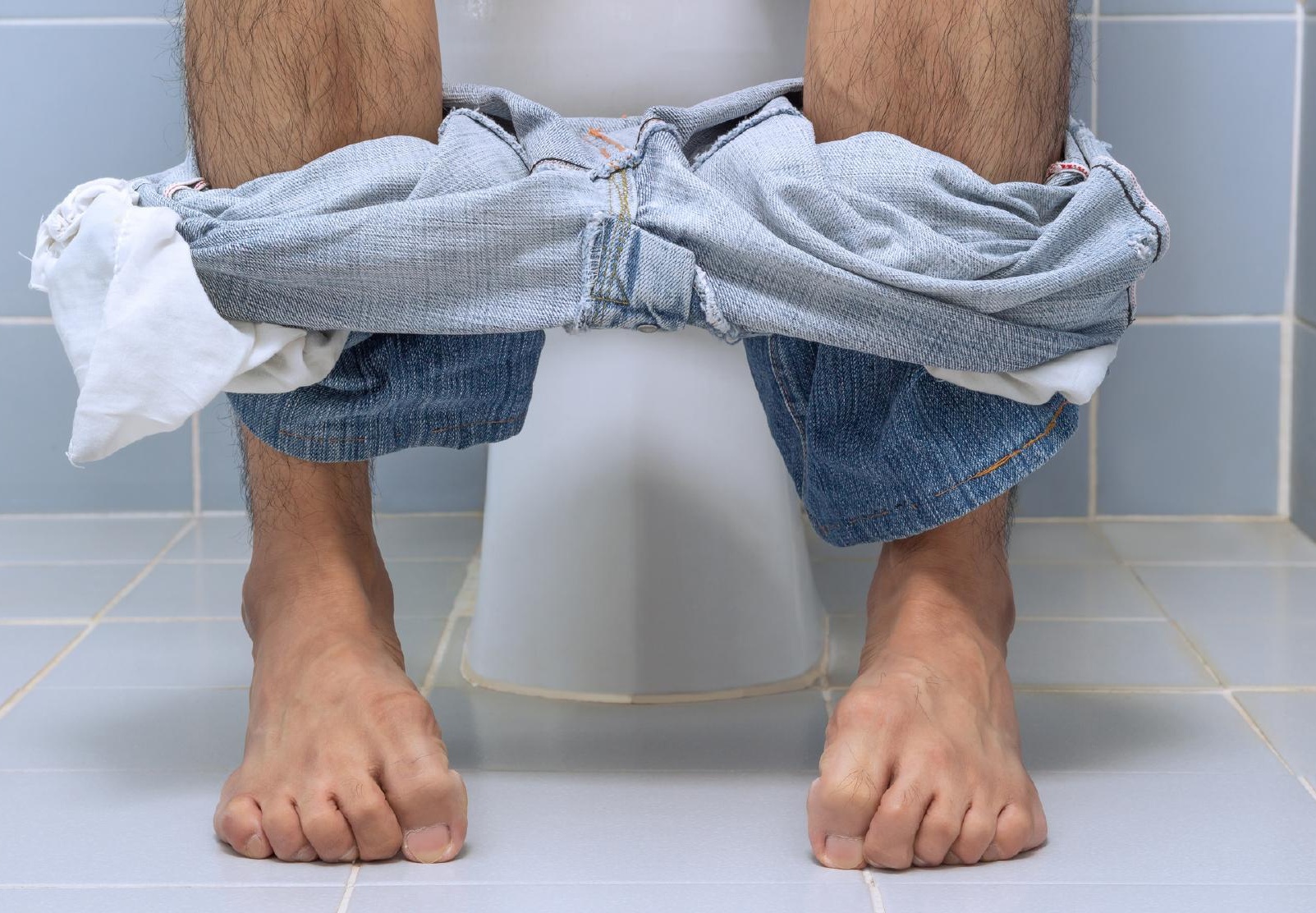




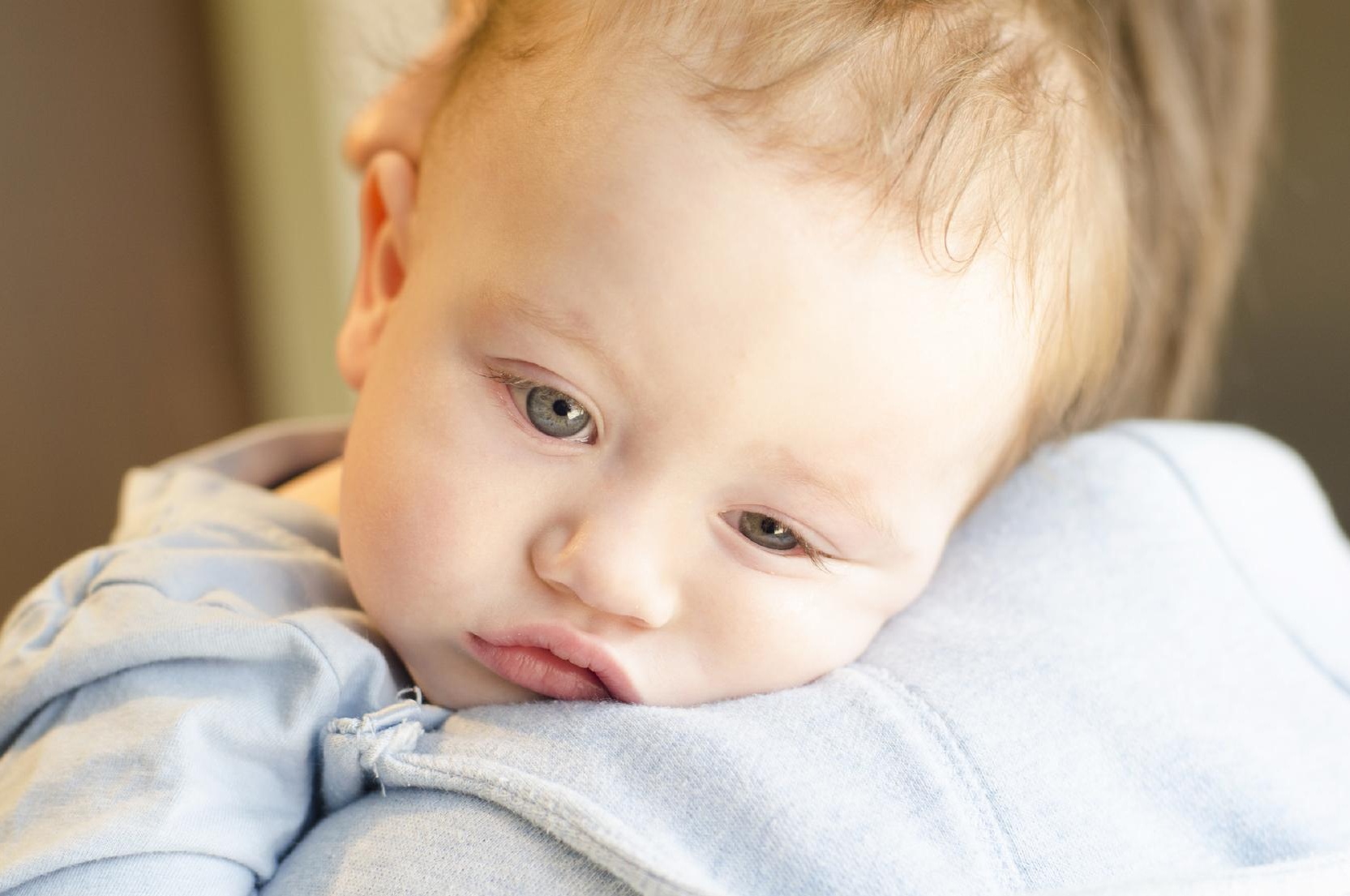

